\title{
Amphetamine primes enhanced motivation toward uncertain choices in rats with genetic alcohol preference
}

\section{Oinio, Ville}

2018-05

Oinio , V , Sundström , M , Bäckström , P , Uhari-Väänänen , J , Kiianmaa , K, Raasmaja , A \& Piepponen , P 2018 , ' Amphetamine primes enhanced motivation toward uncertain choices in rats with genetic alcohol preference ', Psychopharmacology, vol. 235 , no. 5 , pp. 1361-1370 . https://doi.org/10.1007/s00213-018-4847-2

http://hdl.handle.net/10138/311373

https://doi.org/10.1007/s00213-018-4847-2

acceptedVersion

Downloaded from Helda, University of Helsinki institutional repository.

This is an electronic reprint of the original article.

This reprint may differ from the original in pagination and typographic detail.

Please cite the original version. 


\section{Amphetamine primes enhanced motivation toward uncertain choices in rats with genetic alcohol preference}

Ville Oinio1,2, MSc (Pharm), Mikko Sundström1, MSc (Pharm), Pia Bäckström2, PhD , Johanna Uhari-Väänänen1,2,

MSc (Pharm); Kalervo Kiianmaa2, PhD; Atso Raasmaja1, PhD; Petteri Piepponen1, PhD

1Department of Pharmacology and Pharmacotherapy, Faculty of Pharmacy, P.O. Box 56, 00014 University of Helsinki, Helsinki, Finland

2Department of Health, National Institute for Health and Welfare, P.O. Box 30, 00271 Helsinki, Finland

Corresponding author: Ville Oinio, Department of Pharmacology and Pharmacotherapy, Faculty of Pharmacy, P.O. Box 56, 00014 University of Helsinki, Helsinki, Finland

Tel.: +358-(0)294 1911

Fax: +358-(0)2941 59138

E-mail: ville.oinio@helsinki.fi

Acknowledgements

This study was supported by the Finnish Foundation for Alcohol Studies and Orion Research Foundation. The authors declare that they do not have any conflicts of interest related to the data presented in this manuscript. 


\begin{abstract}
Rationale

Comorbidity with gambling disorder (GD) and alcohol use disorder (AUD) is well documented.
\end{abstract}

\title{
Objective
}

The purpose of our study was to examine the influence of genetic alcohol drinking tendency on reward-guided decision making behavior of rats and the impact of dopamine releaser D-amphetamine on this behavior.

\section{Methods}

In this study, Alko alcohol (AA) and Wistar rats went through long periods of operant lever pressing training where the task was to choose the profitable of two options. The lever choices were guided by different-sized sucrose rewards (one or three pellets), and the probability of gaining the larger reward was slowly changed to a level where choosing the smaller reward would be the most profitable in the long run. After training, rats were injected (s.c.) with dopamine releaser D-amphetamine $(0.3,1.0 \mathrm{mg} / \mathrm{kg})$ to study the impact of rapid dopamine release on this learned decision making behavior.

Results

Administration of D-amphetamine promoted unprofitable decision making of AA rats more robustly when compared to Wistar rats. At the same time, D-amphetamine reduced lever pressing responses. Interestingly, we found that this reduction in lever pressing was significantly greater in Wistar rats than in AA rats and it was not linked to motivation to consume sucrose.

\section{Conclusions}

Our results indicate that conditioning to the lever pressing in uncertain environments is more pronounced in AA than in Wistar rats and indicate that the reinforcing effects of a gambling-like environment act as a stronger conditioning factor for rats that exhibit a genetic tendency for high alcohol drinking. 


\section{Introduction}

Comorbidity with gambling disorder (GD, DSM-5) and alcohol use disorder (AUD) is well documented (Slutske et al. 2000; Mann et al. 2017). Twin and adoption studies provide strong evidence of genetics in the etiology of alcoholism (heritability estimates of 40-60\%; e.g., Prescott and Kendler 1999; Prescott et al. 1999; Kampov-Polevoy et al. 2003) and additionally show that GD and AUD have shared genetic vulnerability (Slutske et al. 2000; Slutske et al. 2013). Studies have shown a clear etiological relationship between GD and other addictions (Blanco et al. 2015; Flórez et al. 2016) and they have been found to frequently co-occur (Cowlishaw et al. 2014). One of the common co-occurring addictions in GD patients is AUD, and its prevalence is five to six times higher in disordered gamblers versus the general population (Bischof et al. 2013; Flórez et al. 2016). There is also a positive association between gambling severity and alcohol consumption (French et al. 2008).

Poorly functioning executive cognitive processes that control decision making via functioning of dopamine systems are profoundly linked to GD, as well as to many other psychiatric conditions (Winstanley and Floresco 2016). Changes in dopamine transmission alter reward prediction, causing difficulties in decision making between different value choices (Schultz et al. 1997; Floresco 2016). Dopamine transmission also alters the way an individual processes the "wanting" and "liking" of rewards (Peciña and Berridge 2013; Linnet 2014; Berridge and Kringelbach 2015). These two mechanisms have their specific impact on reward-guided decision making and thus have a significant role in mediating gambling behavior. Also, findings that administer amphetamine enhance gambling urges in pathological gamblers (Zack and Poulos 2004), and chronic gambling-like conditions of uncertain reinforcement act as a promoter of Damphetamine sensitization for rats (Singer et al. 2012; Zack et al. 2014) support the view that excessive dopamine function is a significant promoter of harmful gambling behavior. It has been also shown that alcohol-dependent individuals exert riskier decision making patterns than control groups in various decision making tasks (Brevers et al. 2014; Zois et al. 2014). Reinforcing effects of alcohol are thought to be caused by its effects on cerebral neuronal reward circuitry, through which other drugs of abuse, as well as natural rewards (e.g., drinking, feeding, and sexual behavior) and gambling, induce reinforcement (Wise and Bozarth 1987; Koob and Bloom 1988). Therefore, high sensitivity of these mechanisms to different stimuli might be a factor that predisposes an individual to drug abuse (Honkanen et al. 1999) or gambling. 
Alcohol-preferring Alko alcohol (AA) rats provide a useful tool for identifying an integrative neurobiological factor for GD and AUD. The AA rat line was produced by selective breeding based on high voluntary alcohol consumption, and therefore, these rats are naturally prone to high alcohol consumption (Eriksson 1968; Sinclair et al. 1989; Sommer et al. 2006). AA rats learn quickly to self-administer alcohol without any initiation or shaping, and this alcohol preference is hypothesized to occur due to abnormal function of opioidergic mechanisms (Hyytiä and Sinclair 1989; Koistinen et al. 2001), which are in light of the present knowledge closely related to dopamine functioning (Berridge and Kringelbach 2015). The use of AA rats allows us to examine how those same neuronal mechanisms that are controlling alcohol drinking contribute to the gambling-like behavior of these rats.

Our experiment conducted now is based on and follows up our previous findings with AA rats' reward-guided decision making (Oinio et al. 2017). This experiment allows us to compare three different aspects of reward-guided decision making of rats. The first is rational decision making where animals learn to make the most profitable choice based purely on reward quantity. The second issue examined in the experiment is decision making during long-term probabilistic discounting, which represents risk aversive behavior of the animals. The third issue examined is the effect of pharmacological manipulations of this learned risk aversion. The aim of the study was to examine strain-specific differences between AA and Wistar rats in these three decision making situations. The AA rats were chosen to represent a population that has a genetic tendency for high alcohol consumption, and Wistar rats were chosen to represent a "normal" heterogenic population. The pharmacological manipulations were carried out by administering a dopamine releaser, D-amphetamine, in two different manners. The hypothesis of the study was that stimulation with Damphetamine increases risky decision making behavior of AA rats more than Wistar rats and the short-term sensitization for effects of D-amphetamine would be more pronounced in the AA rats than in Wistar rats.

\section{Material and methods}

Animals

One group $(\mathrm{n}=15)$ of male alcohol-preferring AA rats (National Institute for Health and Welfare, Helsinki, Finland and University of Helsinki, Helsinki, Finland) and one group $(n=16)$ of male Wistar rats were used. At the beginning of the experiment, rats were 3 to 4 months old. On arrival, rats were given 1 week to acclimate to the environment. Food (regular chow SDS RM1 [E] SQC; Witham, Essex, England) and water were available ad libitum in the home cage. 
Rats were housed three per cage in a temperature and humidity-controlled room, with lights controlled on a reversed light/dark cycle of 12/12 h. All experiments were conducted in the dark phase of the light cycle. All testing was in accordance with the Animal Experiment Board of Finland.

Drugs

The dopamine releaser D-amphetamine sulfate (Sigma-Aldrich) was used as the test drug. Doses of D-amphetamine were 0.3 and $1.0 \mathrm{mg} / \mathrm{kg}$ (doses were chosen based on our previous findings, Oinio et al. 2017). Drug doses were calculated as salt weights and dissolved in $0.9 \%$ saline. Saline $(0.9 \%)$ was used also as a vehicle for injection. Drugs and vehicle were given in a Latin square design. Drug doses were administered as s.c. injections at a volume of $1 \mathrm{ml} / \mathrm{kg}$ 20 min prior to testing. Each drug/vehicle test day was preceded by at least three drug-free days. A stable baseline of operant behavior was required for three consecutive days before the next injection was administered. Each rat had their baseline values calculated separately, and each rat also received drugs based on their individual stability in baseline values. The criterion for stable baseline was achieved when the standard error of the mean in LL ("large-lucky," defined later) lever choices $( \pm$ SEM) of three previous baseline session averages was under 5.00.

After three injections were administered, additional test was conducted. In this test, a D-amphetamine dose of $0.3 \mathrm{mg} / \mathrm{kg}$ was given three consecutive days, 20 min prior to placing rats into the operant chambers. On the fourth day, the rats received one dose of D-amphetamine $1.0 \mathrm{mg} / \mathrm{kg} 20 \mathrm{~min}$ prior to testing.

Apparatus

Behavioral testing was conducted in operant chambers $(30.5 \times 24 \times 21 \mathrm{~cm}$; Med-Associates, St Albans, VT, USA) enclosed in sound-attenuating wooden boxes. The boxes were equipped with a fan that provided ventilation and masked extraneous noise. Each chamber was fitted with two retractable levers, one located on each side of a central food tray where sucrose reinforcement (45 mg; Opend, Denmark) was delivered by a pellet dispenser. Above each lever was a cue light. The chambers were illuminated by a single $100 \mathrm{~mA}$ house light located in the top center of the wall opposite the levers. 


\section{Lever press training}

Three days before the first lever press training session, rats were placed in the operant chambers for 15 min each day with the food tray in the chamber containing nine sucrose reward pellets. After this, the rats were returned to their home cage and approximately 30 sucrose pellets were given per cage. This procedure was done to habituate rats to the operant chamber environment and the taste of sucrose.

After habituation days, the training period, which included three phases (A, B, and C), was initiated. In phase A, the rats were trained in forced choice for $60 \mathrm{~min}$ so that only one lever was constantly present (left or right). By pressing the lever, rats received one sucrose pellet with a 3-s time-out during which the cue light was on. Phase A consisted of a total of six training sessions, and the presented lever was changed each session.

In phase B, the rats were trained for 30 min so that only one lever was present (left or right) at the start of the session. Rats received one sucrose pellet for each press. After each press, the lever that was pressed retracted and other lever was presented after 3-s time-out. During time-out, the cue light was lit above the lever that had been pressed and stayed on for $3 \mathrm{~s}$. Phase B consisted of a total of six training sessions.

In the last training phase, $\mathrm{C}$, the rats were trained in a free choice task for 15 min so that both levers were presented at the same time, and by pressing either one of the levers, the rat received one sucrose pellet, the cue light above the lever pressed lit for $15 \mathrm{~s}$, both levers were retracted, and they were presented again after $15 \mathrm{~s}$. Phase $\mathrm{C}$ consisted of a total of six training sessions.

All sessions were conducted in darkness. The house light was on after and before each session but was off during sessions. 


\section{Rational decision making task}

This task was modified from its original form based on the literature (Cardinal and Howes 2005; Adriani and Laviola 2006; St Onge and Floresco 2009) and our previous experiment (Oinio et al. 2017). The rational choice task consisted of a total of 15 sessions, 1 session per day for 5 days a week. At the beginning of the session, rats were placed in the operant chambers, where the house light was on and both levers were retracted. When the session started, the house light went off and both levers were presented simultaneously. One lever was designated the SS-lever ("small/sure"), the other the LL-lever ("large/lucky"). Choice of the SS-lever always delivered one pellet with probability of 100\%, and choice of the LL-lever delivered three pellets with probability of $100 \%$. After pressing a lever, both levers retracted and the cue light above the lever that had been pressed was lit and one (SS lever) or three (LL lever) sucrose pellets were delivered to the food tray. Multiple pellets were delivered $0.5 \mathrm{~s}$ apart. After sucrose was delivered, the cue light remained on for another $15 \mathrm{~s}$, after which both levers were presented again.

In one session, rats had free choice to press the levers at 15 -s intervals and duration of one session was set to 24 lever presses or $30 \mathrm{~min}$. Session durations were registered from all sessions. Based on the literature and our previous findings (Floresco et al. 2008; Haluk and Floresco 2009; Stopper et al. 2013; Oinio et al. 2017), levers were not randomized but were counterbalanced so that for each rat, the LL-lever was designated to be the side that the rat did not spontaneously prefer during training phase C. Designated levers remained consistent throughout sessions for each rat.

The criterion for rational choice behavior [rational choice criterion (RCC)] was set to LL-lever choice of $\geq 75 \%$ or three times the starting level (session 1). Either one of these criteria had to be achieved at least until the 15th session. Rats that achieved either one of these criteria were considered to behave rationally and proceeded to the probabilistic discounting task.

The probabilistic discounting task

This task was divided into three different probability levels where probability of gaining three sucrose pellets by pressing the LL-lever was decreased over time $(50,33,25 \%)$ while the SS-lever always delivered one pellet with $100 \%$ probability. Rats received five consecutive sessions with LL-lever probability of $50 \%$, after that five consecutive sessions with LL-lever probability of $33 \%$ and finally 10 consecutive sessions with LL-lever probability of $25 \%$, 
respectively. After the tenth session at the probability level of $25 \%$, rats were given two saline injections (s.c.) to habituate the rats to the upcoming drug challenges.

\section{Initiation of drug challenges}

The criterion to initiate the drug challenges [risk aversion criterion (RAC)] was set so that the LL-lever choice of rats had to be $\leq 50 \%$ at least after the 10 th session at the probability level of $25 \%$. In addition, completion of all the 24 lever presses during 30 min was required. During the drug challenges, rats went through one session each day at the probability level of $25 \%$ and rats were given injections in the previously described manner.

Satiety control

After drug challenges, the effect of D-amphetamine to the sucrose pellet eating was studied in a 30-min free sucrose pellet eating test. In this test, rats were placed in the operant chamber for $30 \mathrm{~min}$ (house light off) with the additional food cup placed in front of the central food tray. The food cup was filled with $10.0 \mathrm{~g}$ of sucrose pellets. After $30 \mathrm{~min}$, rats were removed and the remaining sucrose pellets were weighed in order to calculate sucrose pellet consumption. Sucrose consumption was calculated in grams per kilograms for each rat. Effects of D-amphetamine doses $(0.3$ and 1.0 $\mathrm{mg} / \mathrm{kg}$ ) were examined, and saline was given as a vehicle. Drugs and vehicle were given s.c. in Latin square design 20 min prior to satiety test with 3-day injection free time between each dose.

Data analysis

Data were analyzed with SPSS version 22.0. Data were collected on all rational choice behavior, probabilistic discounting, drug challenges, lever pressing activity, session duration, and satiety control. Data from rational choice behavior and probabilistic discounting were analyzed with two-way ANOVAs between AA and Wistar rat groups. Data from other experiments were analyzed by within subjects repeated measures ANOVAs followed by Bonferroni's test as a post hoc test. LL-lever choices in the drug challenges task were compared to the vehicle (LL-lever choice $(\%)=$ percentage of LL-lever choices of total lever responses). In addition to the drug-vehicle comparison, the effects of D-amphetamine on LL-lever choices were always compared to the baseline average of the three preceding days. 
Criterion for significance was set at $\mathrm{p}<0.05$. Separate comparisons (only two different values) between AA and Wistar rats were conducted by $\mathrm{t}$ tests or paired $\mathrm{t}$ tests depending on the sample type.

\section{Results}

Rational choice and probabilistic discounting

AA and Wistar rats showed similar decision making behavior during the rational choice and probabilistic discounting tasks (Fig. 1). A two-way ANOVA revealed significant main effects of sessions $[\mathrm{F}(34,770)=25.74 \mathrm{p}<0.001]$ and LLlever choice $(\%)[\mathrm{F}(1,770)=16.4, \mathrm{p}<0.001]$, but no session $\times$ LL-lever choice $(\%)$ interaction was detected $[\mathrm{F}(34$, $770)=1.099, \mathrm{p}=0.3228$ ]. Data presented in the Fig. 1 represent only the behavior of those rats that fulfilled both criteria (RCC and RAC) that were demanded for proceeding to the drug challenges.

Effect of D-amphetamine to the LL-lever choices

In AA rats, a statistically significant increase in LL-lever choices was observed after administration of a single dose of D-amphetamine at a dosage of $1.0 \mathrm{mg} / \mathrm{kg}$ compared to vehicle $[\mathrm{F}(2,22)=9.148, \mathrm{p}<0.01$, post hoc $\mathrm{p}<0.01$ between vehicle and dose $1.0 \mathrm{mg} / \mathrm{kg})]$ or a D-amphetamine dose of $0.3 \mathrm{mg} / \mathrm{kg}[\mathrm{F}(2,22)=9.148, \mathrm{p}<0.01$, post hoc no significance between vehicle and dose $0.3 \mathrm{mg} / \mathrm{kg}$, Fig. 2]. After repeated administration of D-amphetamine, a statistically significant increase in LL-lever choices was observed at the D-amphetamine dose of $1.0 \mathrm{mg} / \mathrm{kg}$ compared to vehicle $[\mathrm{F}(4,44)=7.058, \mathrm{p}<0.01$, post hoc $\mathrm{p}=0.026$ between vehicle and dose $1.0 \mathrm{mg} / \mathrm{kg}]$.

In Wistar rats, no statistically significant main effect on LL-lever choices as observed after administration of a single dose of D-amphetamine at dose of 0.3 or $1.0 \mathrm{mg} / \mathrm{kg}$ compared to vehicle $[\mathrm{F}(2,22)=2.107, \mathrm{p}=0.145$ (one rat missing due zero responses after D-amphetamine dose of $1.0 \mathrm{mg} / \mathrm{kg}$ ), Fig. 2]. After repeated administration of D-amphetamine, no statistically significant main effects on LL-lever choices were observed at the D-amphetamine doses of $0.3 \mathrm{mg} / \mathrm{kg}$ compared to vehicle or D-amphetamine dose of $1.0 \mathrm{mg} / \mathrm{kg}$ compared to vehicle $[\mathrm{F}(4,44)=1.065, \mathrm{p}=0.385$ (one rat missing due zero responses after D-amphetamine dose of $1.0 \mathrm{mg} / \mathrm{kg})]$. 
Effect of D-amphetamine on lever responding

Based on our previous findings (Oinio et al. 2017), D-amphetamine reduced lever responding in AA rats significantly when it was compared to vehicle. Because of this, we explored the lever responses of rats during the drug challenges (Table 1). After administration of D-amphetamine at the dosage of $1.0 \mathrm{mg} / \mathrm{kg}$, a decrease in lever responses was observed in AA and Wistar rats.

Unpaired $\mathrm{t}$ tests between the AA and Wistar rat groups revealed that lever responses were decreased significantly more in Wistar rats than AA rats (Fig. 3). This was the case after single dose administration (unpaired t test $\mathrm{p}<0.05$ ) and as well after repeated dose administration (unpaired t test, $\mathrm{p}<0.05$ ).

Effect of D-amphetamine on the session duration

Session durations were collected from all sessions during the drug challenges (Table 2). At the baseline level (0 mg/kg), Wistar rats were significantly faster than AA to accomplish all the 24 lever presses. This difference was also observed after D-amphetamine doses of $0.3 \mathrm{mg} / \mathrm{kg}$. The highest dose of D-amphetamine caused a major increase in session durations, but these results cannot be trustfully compared because at this dose, many of the rats did not complete all the lever presses, causing the session duration to be the maximum of $30 \mathrm{~min}$ (1800 s).

Effect of D-amphetamine on sucrose pellet consumption

In satiety control, dramatic effects of D-amphetamine on the sucrose eating of AA and Wistar rats were observed (Table 3). Repeated measures ANOVAs revealed significant main effects in both groups $\{[\mathrm{AA}: \mathrm{F}(2,22)=63.920, \mathrm{p}<0.001$, followed by Bonferroni post hoc test results for vehicle and AMPH $0.3 \mathrm{mg} / \mathrm{kg} \mathrm{p}<0.001$, and vehicle and AMPH 1.0 $\mathrm{mg} / \mathrm{kg} \mathrm{p}<0.001)$ ], [Wistar: $\mathrm{F}(2,24)=34.569, \mathrm{p}<0.001$, followed by Bonferroni post hoc test results for vehicle and AMPH $0.3 \mathrm{mg} / \mathrm{kg} \mathrm{p}<0.01$, and vehicle and AMPH $1.0 \mathrm{mg} / \mathrm{kg} \mathrm{p}<0.001)]\}$. 


\section{Discussion}

Here, we studied the effects of D-amphetamine on the reward-guided decision making of AA and Wistar rats. This study aims to find differences in gambling-like decision making between two rat strains where one possesses a genetic tendency for high alcohol consumption. Based on our results, the D-amphetamine promoted more unprofitable decisions and operant lever responding in AA than in Wistar rats, by mechanisms that are not linked solely to the gaining of the reward. These results suggest that the same genetic vulnerabilities that predispose them to a high level of alcohol consumption also creates vulnerability to dopaminergic mechanisms of gambling reinforcement.

As shown numerous times (Cardinal and Howes 2005; St Onge and Floresco 2009; Stopper et al. 2013; Mai et al. 2015) and also discussed in our previous study (Oinio et al. 2017), rats are able to make rational decisions based purely on reward quantity when gaining of the higher reward is guaranteed, but when the element of uncertainty is introduced, rats begin to behave in a risk aversive way. This was also observed here when rats learned to choose the LL-lever over the SS-lever when probability of gaining three pellets was $100 \%$. Right after the probability of gaining three pellets was changed to $50 \%$, rats begun to slowly change lever choices towards the SS-lever, although this would be optimal only when the probability of gaining three pellets is low as $25 \%$. Based on our results, no significant difference was observed in rational choice decision making or decision making during probabilistic discounting between rats that fulfilled criteria that were set to enter drug challenges. Our preliminary data suggest that there might be a slight difference in this baseline behavior when all rats are compared (including those that were excluded due to not fulfilling RCC or RAC), but this must be studied with a larger number of animals than used here.

A dramatic difference, though, is seen after administration of dopamine releaser D-amphetamine. The administration of D-amphetamine increased choices of large but uncertain rewards in AA rats. As predicted, the increase in the LL-lever choices of AA rats after D-amphetamine dose of $1.0 \mathrm{mg} / \mathrm{kg}$ was significant. No significant increase was observed in Wistar rats. After a single D-amphetamine dose of $0.3 \mathrm{mg} / \mathrm{kg}$, we did not observe any increases in LL-lever choices, which was interesting because in our previous study, a D-amphetamine dose of $0.3 \mathrm{mg} / \mathrm{kg}$ caused significant increases in LL-lever choices at probability levels 25 and 20\% (Oinio et al. 2017). Based on the results presented here, we hypothesize that the effect of D-amphetamine dose of $0.3 \mathrm{mg} / \mathrm{kg}$ in the previous study may be due to sensitization for effects of multiple doses of D-amphetamine during the task. This hypothesis was not fully supported by our results from a short series of repeated doses of D-amphetamine at the $0.3 \mathrm{mg} / \mathrm{kg}$ dosage level since repeated doses caused only a 
trend in increases of LL-lever choices compared to vehicle, but this failed to be significant based on the repeated measures ANOVA (paired t tests, however, showed significant differences between vehicle and second D-amphetamine dose $\mathrm{p}<0.05$ and vehicle and third $\mathrm{D}$-amphetamine dose $\mathrm{p}<0.05)$. Another interesting finding was that this increasing trend in LL-lever choices was observed only in AA rats. This indicates that the AA rats could be sensitized to effects of D-amphetamine that promote unprofitable choices. This hypothesis is supported by the findings that during repeated treatment with the dopaminergic drug cocaine, the AA rats developed behavioral (locomotor) sensitization with smaller doses than Wistar rats (Honkanen et al. 1999). The sensitizing effect of cocaine was observed after relatively short series of repeated administrations (four consecutive days) of cocaine, despite the fact that no difference was observed after a single acute treatment with cocaine. This indicates that neuroplastic changes due to high dopaminergic activity occur more rapidly in AA than in Wistar rats. Previous research suggests that this plasticity is located in the striatal regions where enhancement in dopamine release in the nucleus accumbens is seen after four consecutive days of cocaine treatment with AA rats, but not with ANA rats (Mikkola et al. 2001), and indicates that mesolimbic areas in AA rats are extremely prone to sensitization to behavioral effects of dopaminergic drugs. A similar comparison between AA and Wistar rats, however, has not been conducted, but based on our results, it could be hypothesized that there would be a similar difference. Also, as mentioned briefly in our previous report (Oinio et al. 2017), ANA rats would have been intuitively the optimal choice for comparison in reward-guided decision making, but the lack of motivation towards sucrose pellet reward (even after food restriction) prevents us from using ANA rats in this kind of task.

One remarkable finding was the high distribution in LL-lever choices of Wistar rats after the D-amphetamine dose of $1.0 \mathrm{mg} / \mathrm{kg}$. Because of this, we performed a comparison where all rats where viewed separately (see SUPPLEMENT I and II). This comparison revealed that there was high consistency in the LL-lever increasing effect of D-amphetamine among the AA rats, but large inconsistency among the Wistar rats resulting relatively high distribution. This finding could also have a significance in the planning of future studied aiming to find treatment for gambling disorder patients with burden of genetic vulnerability of alcohol use disorder.

Both AA and Wistar rats very willingly consumed a large number of sucrose pellets even under ad libitum feeding of regular chow. Despite this, the D-amphetamine caused significant reduction in sucrose consumption in the satiety test, which was exactly what was predicted based on our previous study (Oinio et al. 2017) and by the literature, because Damphetamine is a drug known to induce anorexic effects (Wise 2006; Melrose et al. 2016; Boekhoudt et al. 2017). It can 
be clearly seen in both strains that D-amphetamine reduced sucrose intake dose dependently, and the highest dose almost abolishes sucrose eating totally. Based on past research showing that in AA rats intake of sweet ingredients activates the same neuronal pathways as alcohol (Dudek et al. 2015), combined with studies showing that AA rats consume high amounts of sweet liquids (Kampov-Polevoy et al. 1999), we predicted that the satiety test would show increased baseline sucrose eating in AA rats compared to Wistar rats, and a more dramatic decrease after Damphetamine in the sucrose eating of Wistar than AA rats. However, this kind of difference between AA and Wistar rats was not detected. A major difference in consumption of sweet was shown between alcohol-preferring rat lines compared to alcohol-avoiding rat lines (Sinclair et al. 1992; Stewart et al. 1994). The sweet liquid consumption of AA and Wistar rats showed a similar pattern of baseline consumption as our present study with sucrose pellets (Sinclair et al. 1992). These two findings do not fully support the view that alcohol-preferring rats are more prone to sweet taste, but indicate that alcohol-avoiding rats have an aversion towards sweet taste.

In addition to reducing sucrose consumption, D-amphetamine also reduced the overall lever responses of Wistar rats significantly more than those of AA rats. When these data are added to the findings from satiety test, it reveals that the differences between the lever responses are very unlikely to be linked to the motivation of gaining sucrose rewards, but more to the lever pressing alone. This indicates that the operant behavior environment combined with the uncertainty acts as a stronger conditioning factor for AA rats than Wistar rats. Because in the task there is no conditioning cue that would be associated to the LL-lever (identical levers are presented simultaneously), only the known outcome of the lever pressing can guide the decision making of the rats. This creates an interesting question about the role of uncertainty and the dopaminergic effects of D-amphetamine in this task. From a gambling point of view, excessive dopaminergic activity is the key focus, because it has been shown that as in other addiction disorders, gambling induces dopamine release in the mesolimbic areas (Joutsa et al. 2012; Linnet 2013). Although the exact role of this dopamine releasing function in the development of gambling disorder is not precisely known, it could be postulated to be linked to the similar neuroplasticity mechanism of the striatal regions that are proposed to be one of the main mechanisms through which substance use addictions are developed (Volkow and Morales 2015). It has been very robustly shown that uncertain events that are linked to the opportunity to get reward increase dopamine release in the striatal regions (Fiorillo et al. 2003; Joutsa et al. 2012). It has also been shown that temporal changes occur in the dopamine levels depending on the outcome of the results in the gambling task (Joutsa et al. 2015). Rewards that are higher than expected increase dopamine release, and rewards that are lower than expected decrease dopamine release in the nucleus accumbens area (Schultz 2006; Linnet 2014). The difference between the expected rewards versus the reward outcome 
creates a reward prediction error (RPE) which is coded by dopaminergic neurons in the Nacc (Schultz 2006). The magnitude of dopamine response in the Nacc is hypothesized to be dependent on the difference between the dopamine levels before and right after the reward is gained, and therefore, unexpected positive outcomes are more rewarding than expected positive outcomes. Studies have shown that in rats, solely a long time of exposure to uncertainty in the operant environment can act as a sensitizing factor for effects of D-amphetamine, providing good evidence that just the excitement can act as a conditioning factor via dopaminergic mechanisms (Zack et al. 2014).

Based on our results, we argue that this dopamine response due to the RPE signal leads to creation of neuroplastic changes in the dopaminergic systems of AA that are greater than those in Wistar rats, and thus, every "win" serves as a greater conditioning factor for AA than Wistar rats. After D-amphetamine administration, the dopamine release in the Nacc could drive the lever choices of rats towards the LL-lever because D-amphetamine is known to induce reinstatement of behavior to which animals have been previously conditioned (de Wit and Stewart 1981; Ledford et al. 2003; Odum and Shahan 2004). Data from human studies also support this view because it has been shown that the psychostimulant D-amphetamine increases the drive to gamble in problem gamblers, but not in healthy controls, which indicates that problem gamblers may be hypersensitive to increases in dopamine release (Zack and Poulos 2004; Cocker et al. 2012).

One may question why, if LL-lever choice acts as a conditioning factor, are the rats not choosing the LL-lever more and more even without the D-amphetamine administration. Explanation for this could be found in the PRE signal theory, because based on this theory, positive outcomes induce dopamine release in the Nacc, but negative outcomes decrease it. We presume that this causes a situation where the "wins" cause dopaminergic impact that is large enough to produce neuroplastic changes, but immediate reductions in dopamine release after "loss" prevent rats from pursuing the LLlever. As long as the dopamine release is enhanced by the D-amphetamine, the dopamine levels in the Nacc stay constantly high despite the loss, making rats unable to adjust their decision making based on the lever press outcomes. This creates a situation where the lever pressing becomes more random and shifts the overall LL-lever choosing close to $50 \%$. This is important from the GD point of view, because the more decisions to initiate the gamble are made, the more the gambler is exposed to the features that are considered to be stimuli that condition gambling (including big unexpected wins, near-miss effects, etc. Singer et al. 2014). Based on this, it could be postulated that high dopamine response due to the PRE signal after wins disturbs the evaluation of the outcome of the next event, but at the same time 
drives gamblers to initiate a new gambling session in those individuals who are conditioned to gambling stimuli. This hypothesis is supported by a human study showing that betting tendencies of subjects with background in pathological gambling is regulated by the pay-offs of consecutive gambling sessions, which is restored to the same level as in healthy controls by a low dose of D2 receptor antagonist haloperidol (Tremblay et al. 2011). This suggests that one reason for the individual differences in gambling behavior between "gambler subtypes" could be the difference in basal dopamine levels in the ventral striatal areas. Based on the RPE signal theory, it is justified to assume that similar outcomes of the gambling session can cause relatively different-sized dopamine responses between different individuals. Modulation of basal dopamine levels could also be a target for individually designed pharmacotherapies where the dose adjustment could be titrated based on individual dopamine levels.

Our findings support the view of heightened reinforcing effects of gambling-like environment in rats with a genetic tendency towards high alcohol consumption and serve as an important platform for studies in the future. We must acknowledge that there might be some unmeasurable variables that may have impact on the differences between the behavior of individual animals. Thus, more specific studies should be conducted to reveal the precise neurobiological differences between these two rat lines that correspond for the behavioral differences found here. This could create possibilities to find specialized pharmacological treatments for GD subtypes that co-occur with AUD.

\section{References}

Adriani W, Laviola G (2006) Delay aversion but preference for large and rare rewards in two choice tasks: implications for the measurement of self-control parameters. BMC Neurosci 7(1):52.

Berridge KC, Kringelbach ML (2015) Pleasure systems in the brain. Neuron 86(3):646-664

Bischof A, Meyer C, Bischof G, Kastirke N, John U, Rumpf HJ (2013) Comorbid Axis I-disorders among subjects with pathological, problem, or at-risk gambling recruited from the general population in Germany: results of the PAGE study. Psychiatry Res 210(3):1065-1070. 
Blanco C, Hanania J, Petry NM, Wall MM, Wang S, Jin CJ, Kendler KS (2015) Towards a comprehensive developmental model of pathological gambling. Addiction 110(8):1340-1351.

Boekhoudt L, Roelofs TJM, de Jong JW, de Leeuw AE, Luijendijk MCM, Wolterink-Donselaar IG, van der Plasse G, Adan RAH (2017) Does activation of midbrain dopamine neurons promote or reduce feeding? Int J Obes 41(7):11311140 .

Brevers D, Bechara A, Cleeremans A, Kornreich C, Verbanck P, Noël X (2014) Impaired decision-making under risk in individuals with alcohol dependence. Alcohol Clin Exp Res 38(7):1924-1931.

Cardinal RN, Howes NJ (2005) Effects of lesions of the nucleus accumbens core on choice between small certain rewards and large uncertain rewards in rats. BMC Neurosci 6(1):37.

Cocker PJ, Dinelle K, Kornelson R, Sossi V, Winstanley CA (2012) Irrational choice under uncertainty correlates with lower striatal D2/3receptor binding in rats. J Neurosci 32(44):15450-15457.

Cowlishaw S, Merkouris S, Chapman A, Radermacher H (2014) Pathological and problem gambling in substance use treatment: a systematic review and meta-analysis. J Subst Abus Treat 46(2):98-105.

de Wit H, Stewart J (1981) Reinstatement of cocaine-reinforced responding in the rat. Psychopharmacology 75(2):134143. 
Dudek M, Abo-Ramadan U, Hermann D, Brown M, Canals S, Sommer WH, Hyytiä P (2015) Brain activation induced by voluntary alcohol and saccharin drinking in rats assessed with manganese-enhanced magnetic resonance imaging. Addict Biol 20(6):1012-1021.

Eriksson K (1968) Genetic selection for voluntary alcohol consumption in the albino rat. Science 159(3816):739-741.

Fiorillo CD, Tobler PN, Schultz W (2003) Discrete coding of reward probability and uncertainty by dopamine neurons. Science 299(5614):1898-1902.

Floresco SB (2016) Dopamine neurons, input integration, and reward prediction errors: E pluribus Unum. Neuron 91(6):1192-1194.

Floresco SB, Block AE, Tse MTL (2008) Inactivation of the medial prefrontal cortex of the rat impairs strategy setshifting, but not reversal learning, using a novel, automated procedure. Behav Brain Res 190(1):85-96.

Flórez G, Saiz PA, Santamaría EM, Álvarez S, Nogueiras L, Arrojo M (2016) Impulsivity, implicit attitudes and explicit cognitions, and alcohol dependence as predictors of pathological gambling. Psychiatry Res 245:392-397.

French MT, Maclean JC, Ettner SL (2008) Drinkers and bettors: investigating the complementarity of alcohol consumption and problem gambling. Drug Alcohol Depend 96(1-2):155-164.

Haluk DM, Floresco SB (2009) Ventral striatal dopamine modulation of different forms of behavioral flexibility. Neuropsychopharmacology 34(8):2041-2052. 
Honkanen A, Mikkola J, Korpi ER, Hyytiä P, Seppälä T, Ahtee L (1999) Enhanced morphine and cocaine-induced behavioral sensitization in alcohol-preferring AA rats. Psychopharmacology 142(3):244-252

Hyytiä P, Sinclair JD (1989) Demonstration of lever pressing for oral ethanol by rats with no prior training or ethanol experience. Alcohol 6(2):161-164.

Joutsa J, Johansson J, Niemelä S, Ollikainen A, Hirvonen MM, Piepponen P, Arponen E, Alho H, Voon V, Rinne JO, Hietala J, Kaasinen V (2012) Mesolimbic dopamine release is linked to symptom severity in pathological gambling. NeuroImage 60(4):1992-1999

Joutsa J, Voon V, Johansson J, Niemelä S, Bergman J, Kaasinen V (2015) Dopaminergic function and intertemporal choice. Transl Psychiatry 5(1):e491.

Kampov-Polevoy AB, Garbutt JC, Janowsky DS (1999) Association between preference for sweets and excessive alcohol intake: a review of animal and human studies. Alcohol Alcohol 34(3):386-395

Kampov-Polevoy AB, Garbutt JC, Khalitov E (2003) Family history of alcoholism and response to sweets. Alcohol Clin Exp Res 27(11):1743-1749.

Koistinen M, Tuomainen P, Hyytiä P, Kiianmaa K (2001) Naltrexone suppresses ethanol intake in 6 hydroxydopaminetreated rats. Alcohol Clin Exp Res 25(11):1605-1612

Koob G, Bloom F (1988) Cellular and molecular mechanisms of drug dependence. Science 242(4879):715-723. 
Ledford CC, Fuchs RA, See RE (2003) Potentiated reinstatement of cocaine-seeking behavior following Damphetamine infusion into the basolateral amygdala. Neuropsychopharmacology 28(10):1721-1729.

Linnet J (2013) The Iowa gambling task and the three fallacies of dopamine in gambling disorder. Front Psychol 4:709

Linnet J (2014) Neurobiological underpinnings of reward anticipation and outcome evaluation in gambling disorder.

Front Behav Neurosci 8:100

Mai B, Sommer S, Hauber W (2015) Dopamine D1/D2 receptor activity in the nucleus accumbens core but not in the nucleus accumbens shell and orbitofrontal cortex modulates risk-based decision making. Int J Neuropsychopharmacol 18:1-9

Mann K, Lemenager T, Zois E, Hoffmann S, Nakovics H, Beutel M, Vogelgesang M, Wölfling K, Kiefer F, FauthBühler M (2017) Comorbidity, family history and personality traits in pathological gamblers compared with healthy controls. Eur Psychiatry 42:120-128.

Melrose AJ, Bailer U, Wierenga CE, Bischoff-Grethe A, Paulus MP, Kaye WH (2016) Psychiatry research : neuroimaging amphetamine alters neural response to sucrose in healthy women. Psychiatry Res Neuroimaging 252:1925.

Mikkola JA, Honkanen A, Piepponen TP, Kiianmaa K, Ahtee L (2001) Effects of repeated cocaine treatment on striatal dopamine release in alcohol-preferring AA and alcohol-avoiding ANA rats. Naunyn Schmiedeberg's Arch Pharmacol 363(2):209-214.

Odum AL, Shahan TA (2004) D-Amphetamine reinstates behavior previously maintained by food: importance of context. Behav Pharmacol 15(7):513-516. 
Oinio V, Bäckström P, Uhari-Väänänen J, Raasmaja A, Piepponen P, Kiianmaa K (2017) Dopaminergic modulation of reward-guided decision making in alcohol-preferring AA rats. Behav Brain Res 326:87-95.

Peciña S, Berridge KC (2013) Dopamine or opioid stimulation of nucleus accumbens similarly amplify cue-triggered 'wanting' for reward: entire core and medial shell mapped as substrates for PIT enhancement. Eur J Neurosci 37(9):1529-1540.

Prescott CA, Kendler KS (1999) Genetic and environmental contributions to alcohol abuse and dependence in a population-based sample of male twins. Am J Psychiatry 156(1):34-40.

Prescott CA, Aggen SH, Kendler KS (1999) Sex differences in the sources of genetic liability to alcohol abuse and dependence in a population-based sample of U.S. twins. Alcohol Clin Exp Res 23(7):1136-1144.

Schultz W (2006) Behavioral theories and the neurophysiology of reward. Annu Rev Psychol 57(1):87-115.

Schultz W, Dayan P, Montague PR (1997) A neural substrate of prediction and reward. Science 275(5306):1593-1599.

Sinclair JD, Lê AD, Kiianmaa K (1989) The AA and ANA rat lines, selected for differences in voluntary alcohol consumption. Experientia 45(9):798-805.

Sinclair JD, Kampov-Polevoy A, Stewart R, Li TK (1992) Taste preferences in rat lines selected for low and high alcohol consumption. Alcohol 9(2):155-160.

Singer BF, Scott-Railton J, Vezina P (2012) Unpredictable saccharin reinforcement enhances locomotor responding to amphetamine. Behav Brain Res 226(1):340-344. 
Slutske WS, Eisen S, True WR, Lyons MJ, Goldberg J, Tsuang M (2000) Common genetic vulnerability for pathological gambling and alcohol dependence in men. Arch Gen Psychiatry 57(7):666-673.

Slutske WS, Ellingson JM, Richmond-Rakerd LS, Zhu G, Martin NG (2013) Shared genetic vulnerability for disordered gambling and alcohol use disorder in men and women: evidence from a national community-based Australian twin study. Twin Res Hum Genet 16(02):525-534.

Sommer W, Hyytiä P, Kiianmaa K (2006) The alcohol-preferring AA and alcohol-avoiding ANA rats: neurobiology of the regulation of alcohol drinking. Addict Biol 11(3-4):289-309.

St Onge JR, Floresco SB (2009) Dopaminergic modulation of risk-based decision making. Neuropsychopharmacology 34(3):681-697.

Stewart RB, Russell RN, Lumeng L, Li TK, Murphy JM (1994) Consumption of sweet, salty, sour, and bitter solutions by selectively bred alcohol-preferring and alcohol-nonpreferring lines of rats. Alcohol Clin Exp Res 18(2):375-381.

Stopper CM, Khayambashi S, Floresco SB (2013) Receptor-specific modulation of risk-based decision making by nucleus accumbens dopamine. Neuropsychopharmacology 38(5):715-728.

Tremblay AM, Desmond RC, Poulos CX, Zack M (2011) Haloperidol modifies instrumental aspects of slot machine gambling in pathological gamblers and healthy controls. Addict Biol 16(3):467-484.

Volkow ND, Morales M (2015) The brain on drugs: from reward to addiction. Cell 162(4):712-725. 
Winstanley CA, Floresco SB (2016) Deciphering decision making: variation in animal models of effort- and uncertainty-based choice reveals distinct neural circuitries underlying core cognitive processes. J Neurosci 36(48):12069-12079.

Wise RA (2006) Role of brain dopamine in food reward and reinforcement. Philos Trans R Soc Lond Ser B Biol Sci 361(1471):1149-1158.

Wise RA, Bozarth MA (1987) A psychomotor stimulant theory of addiction. Psychol Rev 94(4):469-492.

Zack M, Poulos CX (2004) Amphetamine primes motivation to gamble and gambling-related semantic networks in problem gamblers. Neuropsychopharmacology 29(1):195-207.

Zack M, Featherstone RE, Mathewson S, Fletcher PJ (2014) Chronic exposure to a gambling-like schedule of reward predictive stimuli can promote sensitization to amphetamine in rats. Front Behav Neurosci 8:36

Zois E, Kortlang N, Vollstädt-Klein S, Lemenager T, Beutel M, Mann K, Fauth-Bühler M (2014) Decision-making deficits in patients diagnosed with disordered gambling using the Cambridge gambling task: the effects of substance use disorder comorbidity. Brain Behav 4:484-489 


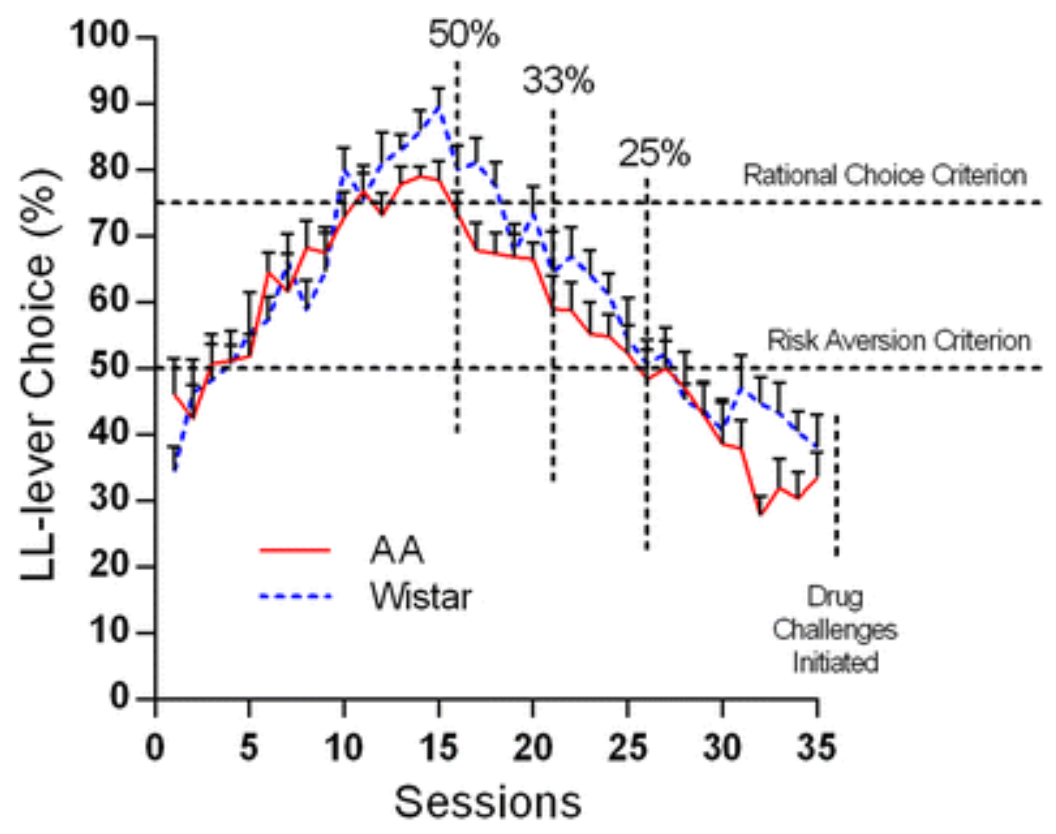

Fig 1. Sessions $1-15$ represent rational choice behavior of Wistar $(n=13)$ and AA rats $(n=12)$ that fulfilled the criteria set for proceeding to drug challenges. Sessions 16 to 20 LL-lever probability 50\%, sessions 21 to 25 LL-lever probability $33 \%$, and from session 26 to session 35 LL-lever probability $25 \%$. [LL-lever choice $(\%)=$ percentage of LLlever choices of total lever responses, \pm SEM]
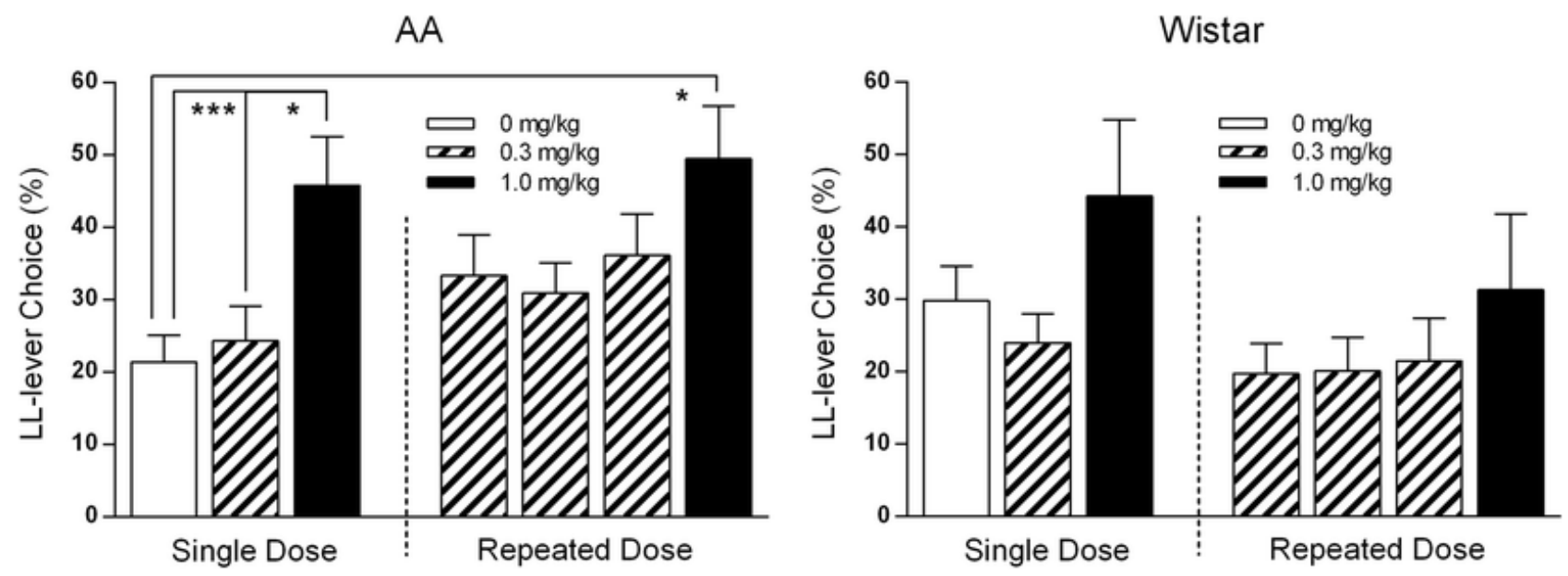

Fig 2. Single dose of D-amphetamine increased LL-lever choices of AA rats at the dose of $1.0 \mathrm{mg} / \mathrm{kg}$ when compared to vehicle or D-amphetamine dose of $0.3 \mathrm{mg} / \mathrm{kg}$. In repeated dose administration, D-amphetamine increased the LL-lever choices after the dose of $1.0 \mathrm{mg} / \mathrm{kg}(\mathrm{n}=12,+\mathrm{SEM})$. Similar increase in LL-lever choices after acute or repeated administration of D-amphetamine was not observed in Wistar rats $(n=13 / 12,+$ SEM $)$ 


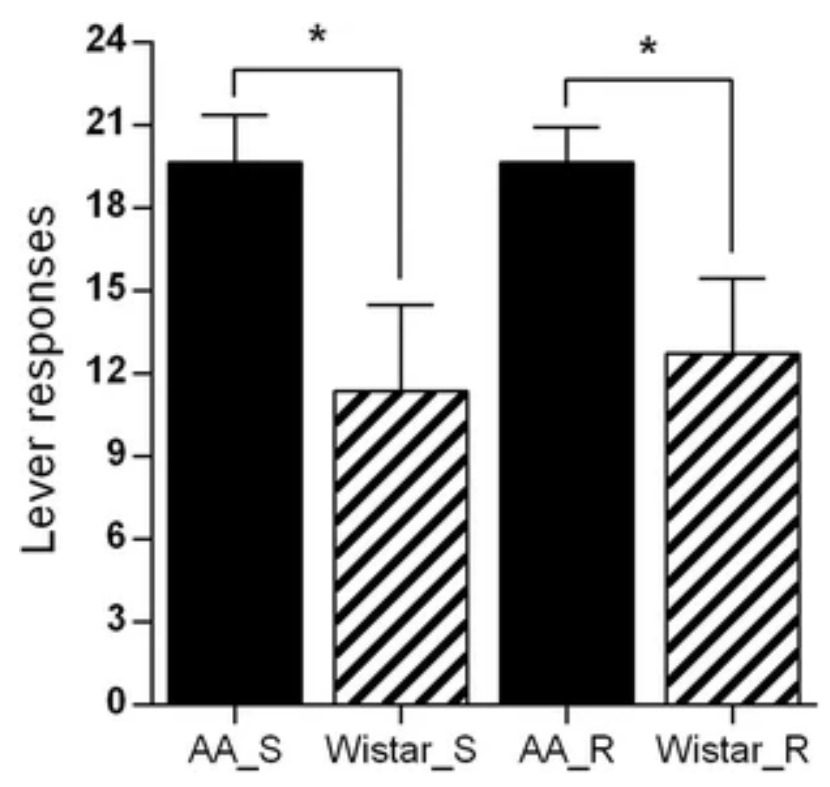

Fig 3. Number of lever responses of AA and Wistar rats during the sessions after single dose (AA_S, Wistar_S) and repeated dose (AA_r, Wistar_r) of D-amphetamine at the dose of $1.0 \mathrm{mg} / \mathrm{kg}$ (n AA 12, Wistar 13/12, + SEM)

Table 1. Effect of D-amphetamine on the lever responses

\begin{tabular}{|l|l|l|}
\hline D-Amphetamine dose (mg/kg) & AA responses & Wistar responses \\
\hline $0 \mathrm{mg} / \mathrm{kg}$ & 24.00 & 24.00 \\
\hline $0.3 \mathrm{mg} / \mathrm{kg}$ & 24.00 & 24.00 \\
\hline $1.0 \mathrm{mg} / \mathrm{kg}$ & $19.67( \pm 1.71)$ & $11.38( \pm 3.13)$ \\
\hline $0.3 \mathrm{mg} / \mathrm{kg} \_1$ & 24.00 & 24.00 \\
\hline $0.3 \mathrm{mg} / \mathrm{kg} \_2$ & 24.00 & 24.00 \\
\hline $0.3 \mathrm{mg} / \mathrm{kg} 3$ & 24.00 & 24.00 \\
\hline $1.0 \mathrm{mg} / \mathrm{kg} \_4$ & $19.67( \pm 1.26)$ & $12.75( \pm 2.73)$ \\
\hline
\end{tabular}
$\mathrm{n}=12 / 13(12)$ 
Table 2. Effect of D-amphetamine on session duration

\begin{tabular}{|c|c|c|c|}
\hline D-Amphetamine dose & AA duration (s) & Wistar duration (s) & t test \\
\hline $0 \mathrm{mg} / \mathrm{kg}$ & $860.42( \pm 128.26)$ & $398.08( \pm 8.48)$ & $* *$ \\
\hline $0.3 \mathrm{mg} / \mathrm{kg}$ & $607.75( \pm 67.75)$ & $444.23( \pm 28.59)$ & * \\
\hline $1.0 \mathrm{mg} / \mathrm{kg}$ & $1176.00( \pm 168.41)$ & $1510.31( \pm 159.28)$ & \\
\hline $0.3 \mathrm{mg} / \mathrm{kg} \_1$ & $843.58( \pm 105.99)$ & $526.58( \pm 43.92)$ & * \\
\hline $0.3 \mathrm{mg} / \mathrm{kg} \_2$ & $797.83( \pm 124.74)$ & $577.33( \pm 103.53)$ & \\
\hline $0.3 \mathrm{mg} / \mathrm{kg} \_3$ & $741.92( \pm 79.47)$ & $482.08( \pm 61.61)$ & $*$ \\
\hline $1.0 \mathrm{mg} / \mathrm{kg} \_4$ & $1523.00( \pm 134.17)$ & $1435.00( \pm 173.46)$ & \\
\hline
\end{tabular}

$\mathrm{n}=$ AA 12, Wistar $13 / 12$

$* \mathrm{p}<0.05 ; * * \mathrm{p}<0.01$

Table 3. Effect of D-amphetamine to the sucrose consumption

\begin{tabular}{|l|l|l|}
\hline D-Amphetamine dose & AA sucrose $(\mathbf{g} / \mathbf{k g})$ & Wistar sucrose $(\mathrm{g} / \mathbf{k g})$ \\
\hline $0 \mathrm{mg} / \mathrm{kg}$ & $7.55( \pm 0.81)$ & $9.56( \pm 0.91)$ \\
\hline $0.3 \mathrm{mg} / \mathrm{kg}$ & $2.35( \pm 0.63)^{* * *}$ & $5.38( \pm 0.71)^{*}$ \\
\hline $1.0 \mathrm{mg} / \mathrm{kg}$ & $0.43( \pm 0.17)^{* * *}$ & $1.34( \pm 0.61)^{* * *}$ \\
\hline
\end{tabular}

$\mathrm{n}=12 / 12, \pm \mathrm{SEM}$

$* \mathrm{p}<0.05$ versus vehicle; $* * * \mathrm{p}<0.001$ versus vehicle 